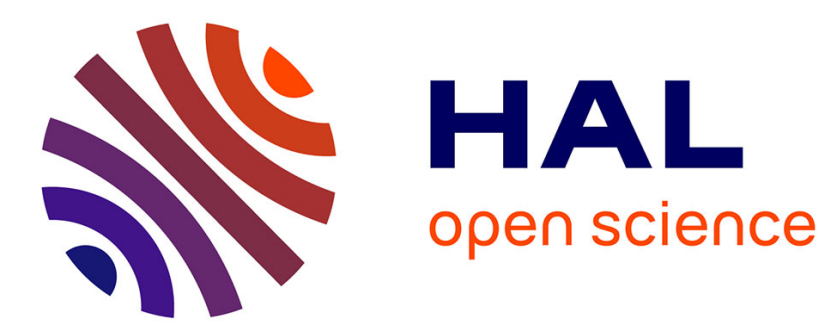

\title{
Closed-form Solutions and Degenerate Cases for Camera Calibration with One-Dimensional Objects
}

\author{
Pär Hammarstedt, Peter Sturm, Anders Heyden
}

\section{To cite this version:}

Pär Hammarstedt, Peter Sturm, Anders Heyden. Closed-form Solutions and Degenerate Cases for Camera Calibration with One-Dimensional Objects. 10th International Conference on Computer Vision (ICCV '05), Oct 2005, Beijing, China. pp.317-324, 10.1109/ICCV.2005.68 • inria-00524397

\section{HAL Id: inria-00524397 \\ https://hal.inria.fr/inria-00524397}

Submitted on 25 May 2011

HAL is a multi-disciplinary open access archive for the deposit and dissemination of scientific research documents, whether they are published or not. The documents may come from teaching and research institutions in France or abroad, or from public or private research centers.
L'archive ouverte pluridisciplinaire $\mathbf{H A L}$, est destinée au dépôt et à la diffusion de documents scientifiques de niveau recherche, publiés ou non, émanant des établissements d'enseignement et de recherche français ou étrangers, des laboratoires publics ou privés. 


\title{
Degenerate Cases and Closed-form Solutions for Camera Calibration with One-Dimensional Objects
}

\author{
Pär Hammarstedt ${ }^{\dagger}$, Peter Sturm ${ }^{\ddagger}$, Anders Heyden ${ }^{\dagger}$ \\ $\dagger$ Applied Mathematics Group, School of Technology and Society \\ Malmo University, 20506 Malmo, Sweden \\ par.hammarstedt@ts.mah.se, heyden@ts.mah.se \\ † MOVI group, INRIA Rhône-Alpes \\ 38330 Montbonnot St Martin, France \\ peter.sturm@inrialpes.fr
}

\begin{abstract}
Camera Calibration with one-dimensional objects is based on an algebraic constraint on the image of the absolute conic. We will give an alternative derivation to this constraint, allowing a geometrical interpretation. From this we derive the degenerate cases, or critical motions, where the calibration algorithm will fail. We also show that constraints on the intrinsic parameters lead to simplified closed-form solutions and a reduced set of critical motions. A simulation and a real data experiment is performed to evaluate the accuracy of the calibration result for motions close to being critical.
\end{abstract}

\section{Introduction}

In computer vision, metric 3D reconstruction from images requires the camera to be calibrated. The main camera calibration techniques can be classified into five groups. In $3 D$ reference object calibration an object with known geometry is used $[12,3]$. In $2 D$ plane based calibration planar patterns are used $[10,13]$. 1D object calibration is discussed in this paper. The remaining two groups are selfcalibration, where point correspondences between images of an unknown scene are used [7, 6, 5, 3], and motion constrained calibration, where the camera is confined to some special kind of motion $[1,4,8]$. In some cases of camera motion, known as critical motions, the calibration algorithms will fail. This has been studied in detail for $3 D$ reference object calibration in [2] and for self-calibration in [9].

In this paper we aim to complete the theory of 1D object calibration by identifying the critical motions. We show how to reduce them when partial knowledge of the cameras calibration parameters is given. Camera calibration using one-dimensional (1D) objects was recently proposed in [14]. Here, the calibration object consists of a set of at least three collinear points. The motion of the object is constrained by one point being fixed. One advantage of using 1D objects for calibration are that $1 \mathrm{D}$ objects with known geometry are easy to construct. Another advantage is that in a multi-camera environment, all cameras can observe the entire calibration object simultaneously, which is a prerequisite for calibration and hard to obtain with 3- and 2-dimensional calibration objects. In practice, the 1D object can be constructed by marking three points on a stick.

The paper is organized as follows: In Section 2 a brief review of camera calibration with $1 \mathrm{D}$ objects is given. In Section 3 a geometrical interpretation of the calibration constraint is presented, from which the critical motions are identified in Section 4. Section 5 describes how simplified closed-form solutions reduce the critical motions. Section 6 validates the theoretical results by two sets of experiments.

\section{Preliminaries}

\subsection{Notation}

We will use the standard pin-hole camera model:

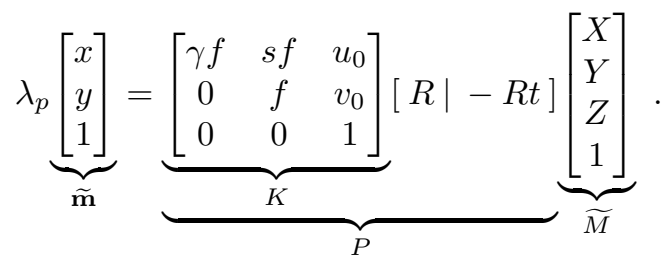


Here, $f$ denotes the focal length, $\gamma$ the aspect ratio, $s$ the skew and $\left(u_{0}, v_{0}\right)$ the principal point. These are called the intrinsic parameters and are contained in the uppertriangular calibration matrix $K$. Furthermore, $R$ and $t$ denote the relation between the camera coordinate system and the object coordinate system, where $R$ is a rotation matrix and $t$ a translation vector, i.e. a Euclidean transformation. $P$ is the camera matrix and $\lambda_{p}$ is the projective depth of $\widetilde{\mathbf{m}}$. A 2D point is denoted by either $\mathbf{m}=[x, y]^{T}$ or $\widetilde{\mathbf{m}}=$ $[x, y, 1]^{T}$. A 3D point is denoted by either $M=[X, Y, Z]^{T}$ or $\widetilde{M}=[X, Y, Z, 1]^{T}$.

\subsection{Camera Calibration with 1D Objects}

We will now give a brief review of the theory for camera calibration with one-dimensional objects, following [14]. In the following, we often call the one-dimensional calibration object a "stick", for simplicity.

Refer to Figure 1 where point $O$ is the camera center. Point $A$ is fixed relative to the camera, and the length of the stick $A B$ is

$$
L=\|B-A\| .
$$

The position of point $C$ is given by

$$
C=\lambda_{A} A+\lambda_{B} B,
$$

where $\lambda_{A}$ and $\lambda_{B}$ are known. Without loss of generality we choose $R=I$ and $t=0$, which implies that the optical center $O$ is at the origin. Let the unknown depths of $A, B$ and $C$ be $z_{A}, z_{B}$ and $z_{C}$, respectively. According to (1) we have $A=z_{A} K^{-1} \widetilde{\mathbf{a}}$ and similarly for $B$ and $C$, so equation (3) gives

$$
z_{C} \widetilde{\mathbf{c}}=z_{A} \lambda_{A} \widetilde{\mathbf{a}}+z_{B} \lambda_{B} \widetilde{\mathbf{b}} .
$$

By performing cross products on both sides of (4) with $\widetilde{\mathbf{c}}$ and scalar products with $(\widetilde{\mathbf{b}} \times \widetilde{\mathbf{c}})$ we obtain

$$
z_{B}=-z_{A} \frac{\lambda_{A}(\widetilde{\mathbf{a}} \times \widetilde{\mathbf{c}}) \cdot(\widetilde{\mathbf{b}} \times \widetilde{\mathbf{c}})}{\lambda_{B}(\widetilde{\mathbf{b}} \times \widetilde{\mathbf{c}}) \cdot(\widetilde{\mathbf{b}} \times \widetilde{\mathbf{c}})} .
$$

From (2) we have

$$
\left\|K^{-1}\left(z_{B} \widetilde{\mathbf{b}}-z_{A} \widetilde{\mathbf{a}}\right)\right\|=L
$$

and by substituting $z_{B}$ by (5) in this equation we get

$$
z_{A}\left\|K^{-1} \mathbf{h}\right\|=L
$$

where

$$
\begin{aligned}
\mathbf{h} & =\left[h_{1}, h_{2}, h_{3}\right]^{T}=\frac{\left(z_{B} \widetilde{\mathbf{b}}-z_{A} \widetilde{\mathbf{a}}\right)}{z_{A}}= \\
& =\widetilde{\mathbf{a}}+\frac{\lambda_{A}(\widetilde{\mathbf{a}} \times \widetilde{\mathbf{c}}) \cdot(\widetilde{\mathbf{b}} \times \widetilde{\mathbf{c}})}{\lambda_{B}(\widetilde{\mathbf{b}} \times \widetilde{\mathbf{c}}) \cdot(\widetilde{\mathbf{b}} \times \widetilde{\mathbf{c}})} .
\end{aligned}
$$

Equation (7) is equivalent to

$$
z_{A}^{2} \mathbf{h}^{T} \omega \mathbf{h}=L^{2}
$$

where

$$
\begin{gathered}
\omega=K^{-T} K^{-1}= \\
=\left[\begin{array}{ccc}
\frac{1}{f^{2} \gamma^{2}} & -\frac{s}{f^{2} \gamma^{2}} & \frac{s y_{0}-x_{0}}{f^{2} \gamma^{2}} \\
-\frac{s}{f^{2} \gamma^{2}} & \frac{s^{2}}{f^{2} \gamma^{2}}+\frac{1}{f^{2}} & -\frac{s\left(s y_{0}-x_{0}\right)}{f^{2} \gamma^{2}}-\frac{y_{0}}{f^{2}} \\
\frac{s y_{0}-x_{0}}{f^{2} \gamma^{2}} & -\frac{s\left(s y_{0}-x_{0}\right)}{f^{2} \gamma^{2}}-\frac{y_{0}}{f^{2}} & \frac{\left(s y_{0}-x_{0}\right)^{2}}{f^{2} \gamma^{2}}+\frac{y_{0}^{2}}{f^{2}}+1
\end{array}\right]
\end{gathered}
$$

is the image of the absolute conic [3]. Let $\omega_{i j}$ be the element of $\omega$ at row $i$ and column $j$. Then $\omega$, which is symmetric, can be defined by

$$
\mathbf{d}=\left[\omega_{11}, \omega_{12}, \omega_{22}, \omega_{13}, \omega_{23}, \omega_{33}\right]^{T} .
$$

With $\mathbf{x}=z_{A}^{2} \mathbf{d}$ and

$$
\mathbf{u}=\left[h_{1}^{2}, 2 h_{1} h_{2}, h_{2}^{2}, 2 h_{1} h_{3}, 2 h_{2} h_{3}, h_{3}^{2}\right]^{T},
$$

equation (10) becomes

$$
\mathbf{u}^{T} \mathbf{x}=L^{2},
$$

giving one constraint on $z_{A}$ and the intrinsic parameters in $K$ per image. In the most general case with six unknowns, we need at least six observations of the stick for calibration.

Given $N$ images, the solution to (10) is found by solving a linear system of one equation per image, such that symmetry of $\omega$ is enforced:

$$
\mathbf{U x}=L^{2} \mathbf{1}
$$

where $\mathbf{U}=\left[\mathbf{u}_{1}, \ldots, \mathbf{u}_{N}\right]^{T}$ and $\mathbf{1}=[1, \ldots, 1]^{T}$. The least squares solution is then given by

$$
\mathbf{x}=L^{2}\left(\mathbf{U}^{T} \mathbf{U}\right)^{-1} \mathbf{U}^{T} \mathbf{1} .
$$

$K$ and $z_{A}$ can then be found by Cholesky decomposition of $z_{A}^{2} \omega$ (which is given by $\mathbf{x}$ ).

\section{Geometrical Interpretation}

In order to identify the critical motions of the stick for which calibration will fail, we will now interpret equation (10) in geometrical terms. Refer to Figure 2. Let the line through $A$ and $B$ be $l_{A B}$. The intersection of $l_{A B}$ and the plane at infinity $\pi_{\infty}$ is given by $X_{\infty}=\widetilde{B}-\widetilde{A}$. Projecting this point onto the image we obtain the vanishing point

$$
\mathbf{v}=\left[v_{1}, v_{2}, v_{3}\right]^{T}
$$

of the line $l_{A B}$ :

$$
\begin{aligned}
\mathbf{v} & =P X_{\infty}=K[I \mid 0](\widetilde{B}-\widetilde{A})=K(B-A) \\
& =z_{B}\left[x_{B}, y_{B}, 1\right]^{T}-z_{A}\left[x_{A}, y_{A}, 1\right]^{T} \\
& =z_{B} \widetilde{\mathbf{b}}-z_{A} \widetilde{\mathbf{a}} .
\end{aligned}
$$




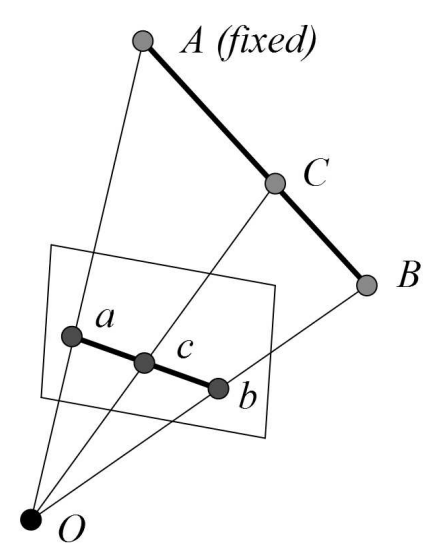

Figure 1. Illustration of 1D calibration objects

Using (8) we have

$$
\mathbf{v}=z_{A} \mathbf{h} .
$$

Alternatively, let $X=B-A$. With $\mathbf{v}=K X$ we get

$$
\begin{gathered}
1=\frac{X^{T} X}{X^{T} X}=\frac{X^{T} K^{T} K^{-T} K^{-1} K X}{\|X\|^{2}}=\frac{\mathbf{v}^{T} \omega \mathbf{v}}{L^{2}} \Rightarrow \\
\mathbf{v}^{T} \omega \mathbf{v}=L^{2},
\end{gathered}
$$

so that (14) holds, since (15) $\Leftrightarrow(10)$. We can now interpret (15) as follows: the algebraic distance between the vanishing point of the stick and the image of the absolute conic equals $L^{2}$.

Notice that for calibration only, the actual length of the stick does not have to be known; using the constraint (10) will give us $z_{A}$ in units of $L$ (i.e. $z_{A}$ will be the unit-less ratio of stick length and the actual metric depth of $A$ ), and always the correct calibration $K$. This is the typical scaledepth ambiguity in reconstruction; a change in scale can be compensated by a change in depth without changing the calibration matrix.

\section{Degenerate cases}

A motion of the stick is critical if and only if (15) has more than one solution. Given a number of observations of the stick, let $\mathbf{v}_{i}$ be the vanishing point in image $i$. The motion is now critical when the vanishing points of the stick $\mathbf{v}_{i}$ lie on a conic $\omega^{\prime}$ so that

$$
\mathbf{v}_{i}^{T} \omega^{\prime} \mathbf{v}_{i}=0 \quad \forall i
$$

since then, if $\omega$ is a solution to (15), $\omega+k \omega^{\prime}, k \in \mathbf{R}$, is also a solution by

$$
\mathbf{v}_{i}^{T}\left(\omega+k \omega^{\prime}\right) \mathbf{v}_{i}=\mathbf{v}_{i}^{T} \omega \mathbf{v}_{i}+k \mathbf{v}_{i}^{T} \omega^{\prime} \mathbf{v}_{i}=L^{2} .
$$

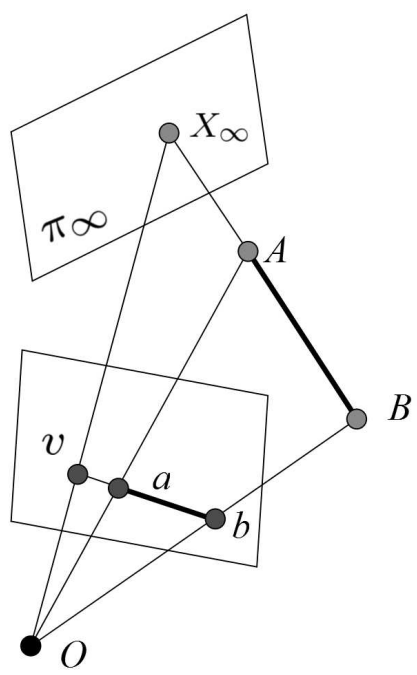

Figure 2. Geometrical interpretation of calibration from 1D objects

When solving for $\omega$ in (13), the actual solution $\omega+k \omega^{\prime}$ is constrained to a symmetric matrix, therefore $\omega^{\prime}$ must also be symmetric. If additional constraints are placed on $\omega$, such that $\omega$ is of a more constrained form, then $\omega^{\prime}$ must also be of the same, more constrained, form. This is done by incorporating knowledge on the intrinsic parameters as will be described in section 5 .

Note that equation (10) would have no solutions (with $L \neq 0$ ) if $\mathbf{v}_{i}$ would lie on $\omega$ such that $\mathbf{v}_{i}^{T} \omega \mathbf{v}_{i}=0$. Since $\omega$ is a virtual conic and the vanishing points are real (from $\mathbf{v}=P(\widetilde{B}-\widetilde{A})$ ), this however only happens if $\mathbf{v}_{i}=0 \forall i$. This corresponds to the uninteresting case where $A$ and $B$ both lie on an optical ray of the camera in all images so that $\mathbf{a}$ and $\mathbf{b}$ coincide.

\subsection{Critical motions}

We now want to identify the critical motions of the stick that give rise to the degenerate cases where the vanishing points lie on a conic $\omega^{\prime}$ in the image plane.

Assume $\mathbf{v}_{i} \omega^{\prime} \mathbf{v}_{i}=0$. Let $D_{i}$ be any point on the stick in image $i$ and $E_{i}=D_{i}-A$ the same point expressed in a coordinate system with origin translated to $A$. With $P=K[I \mid 0]$ and $\mathbf{v}_{i}=K[I \mid 0]\left(\widetilde{D_{i}}-\widetilde{A}\right)=K E_{i}$ we get

$$
\begin{array}{rll}
\mathbf{v}_{i}^{T} \omega^{\prime} \mathbf{v}_{i}=0 & \Leftrightarrow & E_{i}^{T} K^{T} \omega^{\prime} K E_{i}=0 \quad \Leftrightarrow \\
E_{i}^{T} \omega^{\prime \prime} E_{i}=0 & \Leftrightarrow & \widetilde{E_{i}^{T}}\left[\begin{array}{cc}
\omega^{\prime \prime} & 0 \\
0 & 0
\end{array}\right] \widetilde{E_{i}}=0
\end{array}
$$

where $\omega^{\prime \prime}$ is symmetric. Equation (16) tells us that all points 
on the stick in all positions lie on a quadric of rank less than or equal to 3 , in this case a cone, centered at $A$.

In other words: the motion is critical if and only if the vanishing points of the stick lie on a conic $\omega^{\prime}$. Since we deal with perspective projection, this is exactly the case if the stick's point at infinity traces out a conic on the plane at infinity during the motion (which can be a degenerate conic, e.g. consisting of 2 straight lines). This in turn means that the stick, when seen as an infinite line, traces out a cone, with the fixed point $A$ as vertex and the above conic as "generator". Note that the cone does not need to be circular, i.e. the locus of an individual point on the stick does not need to be a planar circle for the degeneracy to occur. Furthermore, as mentioned above, the generating conic may be degenerate, e.g. consisting of 2 straight lines. As for the stick's motion, this means that it is waved in 2 different planes.

Note that critical motions do not depend on the actual position of the stick's fixed point $A$; they only depend on the stick's orientation (and in special cases, see below, on its orientation with respect to the camera).

In [14] some partial results on critical motions are given; the case of a circular cone. This is of course degenerate, but there are many more critical motions, as we have seen.

\subsection{Safe motions}

In practice, all critical motions should of course be avoided. From the above said, we observe that this can be achieved by for example moving the stick in three or more non-parallel planes, which may be realized by some zig-zag motion. Many other examples can be found, e.g. moving the stick in a spiral.

\section{Closed-Form Solutions}

We will now look at the closed-form solutions for the cases where some of the intrinsic parameters of the camera are known and show what degeneracies there are in these cases. We also show that the number of images required for calibration using these closed-form solutions will be smaller than in the general case.

\subsection{Unknown focal length}

Assume that only the focal length of the camera is unknown. The image coordinate system can then be transformed such that $s=0, \gamma=1$ and $\left(x_{0}, y_{0}\right)=(0,0)$. Then

$$
\omega=\left[\begin{array}{ccc}
\frac{1}{f^{2}} & 0 & 0 \\
0 & \frac{1}{f^{2}} & 0 \\
0 & 0 & 1
\end{array}\right]
$$

so that the calibration problem reduces to solving equation (13) where (in the minimal case of only two images)

$$
\mathbf{U}=\left[\begin{array}{ll}
h_{11}^{2}+h_{21}^{2} & h_{31}^{2} \\
h_{12}^{2}+h_{22}^{2} & h_{32}^{2}
\end{array}\right], \quad \mathbf{x}=\left[\begin{array}{c}
z_{A}^{2} \\
f^{2} \\
z_{A}^{2}
\end{array}\right]
$$

and $h_{j i}$ is $h_{j}$ in image $i$. We observe that here, only two images are needed for calibration since then $\mathbf{U}$ is invertible. Modifying the calibration algorithm in this way fixes known camera parameters to their correct value and reduces the set of critical motions to the case where the vanishing points all lie on a circle centered in the image.

This can also be verified by noting that (13) has a unique solution if and only if $\operatorname{det}(\mathbf{U}) \neq 0$. Now, denoting $v_{j}$ in image $i$ by $v_{j i}$,

$$
\begin{array}{cc}
\operatorname{det}(\mathbf{U})=h_{32}^{2}\left(h_{11}^{2}+h_{21}^{2}\right)-h_{31}^{2}\left(h_{12}^{2}+h_{22}^{2}\right)=0 & \Leftrightarrow \\
& v_{32}^{2}\left(v_{11}^{2}+v_{21}^{2}\right)-v_{31}^{2}\left(v_{12}^{2}+v_{22}^{2}\right)=0,
\end{array}
$$

since $\mathbf{v}=z_{A} \mathbf{h}$ (by (14)) and $z_{A} \neq 0$ since all depths are positive. The condition for a critical motion (17) is fulfilled if $v_{3 i}=0 \forall i$, which corresponds to the case where the vanishing point of the stick is a point at infinity so that the stick is moving in a plane parallel to the image plane, or if

$$
\begin{gathered}
\left(\frac{v_{11}}{v_{31}}\right)^{2}+\left(\frac{v_{21}}{v_{31}}\right)^{2}=\left(\frac{v_{12}}{v_{32}}\right)^{2}+\left(\frac{v_{22}}{v_{32}}\right)^{2} \Leftrightarrow \\
v_{x 1}^{2}+v_{y 1}^{2}=v_{x 2}^{2}+v_{y 2}^{2}
\end{gathered}
$$

where $v_{x i}$ and $v_{y i}$ are the $x$ - and $y$-coordinates of the vanishing point in image $i$ (since $\mathbf{v}$ is expressed in homogeneous coordinates), meaning that the vanishing points lie on a centered circle. Now equation (16) gives that the stick lies on a quadric of the form

$$
\left[\begin{array}{llll}
a & 0 & 0 & 0 \\
0 & a & 0 & 0 \\
0 & 0 & b & 0 \\
0 & 0 & 0 & 0
\end{array}\right]
$$

centered at $A$, where $a, b \in \mathbf{R}$, which is a circular cone whose axis of symmetry is parallel to the $\mathrm{z}$ axis (see Figure 3 ). Waving the stick in a plane parallel to the image plane is then also a degenerate motion, since it is a special case of a circular cone (it's like a cone that is squashed to a plane). In this case, the vanishing points of the stick are points at infinity of the image plane. The line at infinity of the image plane is a (degenerate) conic, of the required form (centered circle).

\subsection{Unknown focal length and aspect ratio}

In this case

$$
\omega=\left[\begin{array}{ccc}
\frac{1}{f^{2} \gamma^{2}} & 0 & 0 \\
0 & \frac{1}{f^{2}} & 0 \\
0 & 0 & 1
\end{array}\right]
$$



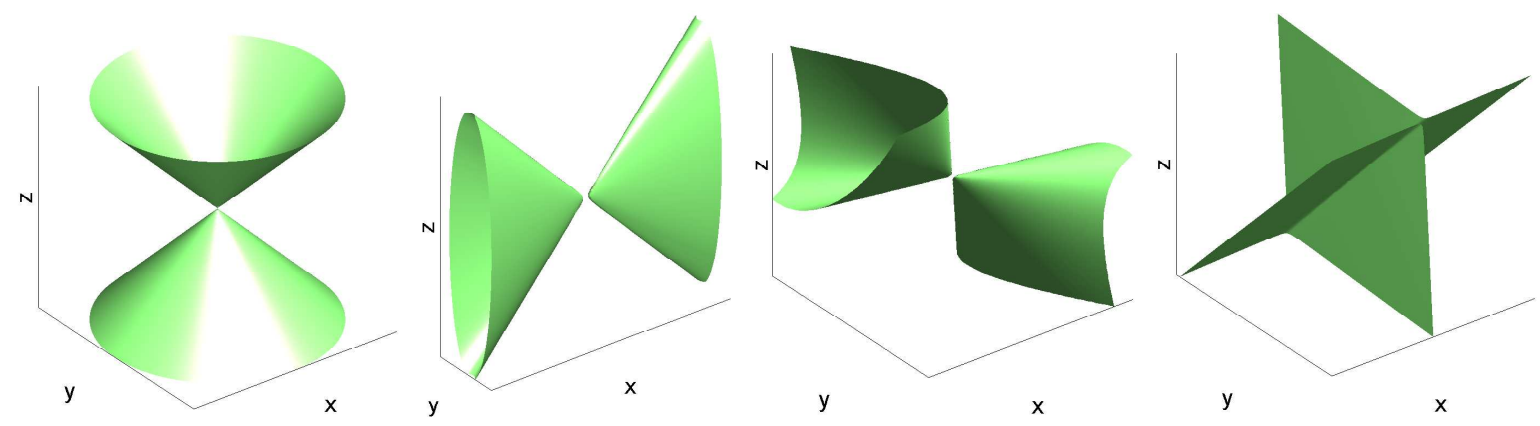

Figure 3. Examples of critical quadric surfaces. If only the focal length is unknown, the critical surface is a circular cone with axis of symmetry is parallel to the $z$ axis (far left). With also the aspect ratio unknown the surface is an elliptical cone with main axis parallel to any two coordinate axes, and axis of symmetry parallel to the third one (center left). Examples of general quadrics representing critical surfaces in the general case (right). The camera has the optical axis coinciding with the z-axis and the image plane coordinate axes coinciding with the $x$ - and $y$-axis

The calibration problem reduces to solving equation (13) where (in the minimal case of three images)

$$
\mathbf{U}=\left[\begin{array}{lll}
h_{11}^{2} & h_{21}^{2} & h_{31}^{2} \\
h_{12}^{2} & h_{22}^{2} & h_{32}^{2} \\
h_{13}^{2} & h_{23}^{2} & h_{33}^{2}
\end{array}\right], \quad \mathbf{x}=\left[\begin{array}{c}
\frac{z_{A}^{2}}{f^{2} \gamma^{2}} \\
\frac{z_{A}^{2}}{f^{2}} \\
z_{A}^{2}
\end{array}\right]
$$

and $h_{j i}$ is $h_{j}$ in image $i$, which has a unique solution if and only if $\operatorname{det}(\mathbf{U}) \neq 0$. Now $\operatorname{det}(\mathbf{U})=0$ if and only if

$$
\begin{gathered}
v_{11}^{2} v_{22}^{2} v_{33}^{2}+v_{21}^{2} v_{32}^{2} v_{13}^{2}+v_{31}^{2} v_{22}^{2} v_{13}^{2}- \\
v_{31}^{2} v_{22}^{2} v_{13}^{2}-v_{21}^{2} v_{12}^{2} v_{33}^{2}-v_{11}^{2} v_{23}^{2} v_{32}^{2}=0
\end{gathered}
$$

which is the condition for a critical motion. It is fulfilled either if $v_{j_{0} i}=0 \forall i$ and for some fixed $j_{0}$, corresponding to a motion of the stick in any of the two image coordinate axis planes $\left(v_{1}=0\right.$ or $\left.v_{2}=0\right)$ or in a plane parallel to the image plane $\left(v_{3}=0\right)$, or (by rewriting (18) by dividing with $v_{31}^{2} v_{32}^{2} v_{33}^{2}$, renaming $\frac{v_{1 i}}{v_{3 i}}$ to $v_{x i}$ and $\frac{v_{2 i}}{v_{3 i}}$ to $v_{y i}$, which then are the image coordinates of the vanishing point) if

$v_{x 1}^{2} v_{y 2}^{2}+v_{y 1}^{2} v_{x 3}^{2}+v_{x 2}^{2} v_{y 3}^{2}-v_{y 2}^{2} v_{x 3}^{2}-v_{y 1}^{2} v_{x 2}^{2}-v_{x 1}^{2} v_{y 3}^{2}=0$.

This means that the vanishing points are on a ellipse centered in the image, with axes coinciding with the image $\mathrm{x}$ and $y$ axes. Equation (16) gives that the stick then moves on the surface of an elliptical cone with main axis parallel to any two coordinate axis, and axis of symmetry parallel to the third one, see Figure 3.

\subsection{Unknown focal length and principal point}

Another frequently occurring condition in camera calibration is that of $s=0$ and $\gamma=1$. In this case we find the simplified closed-form solution by observing that

$$
\omega=\left[\begin{array}{ccc}
\frac{1}{f^{2}} & 0 & -\frac{x_{0}}{f^{2}} \\
0 & \frac{1}{f^{2}} & -\frac{y_{0}}{f^{2}} \\
-\frac{x_{0}}{f^{2}} & -\frac{y_{0}}{f^{2}} & \frac{x_{0}^{2}}{f^{2}}+\frac{y_{0}^{2}}{f^{2}}+1
\end{array}\right] . .
$$

This reduces the problem to solving equation (13) where (in the minimal case of four images)

$$
\begin{aligned}
& \mathbf{U}=\left[\begin{array}{llll}
h_{11}^{2}+h_{21}^{2} & 2 h_{11} h_{31} & 2 h_{21} h_{31} & h_{31}^{2} \\
h_{12}^{2}+h_{22}^{2} & 2 h_{12} h_{32} & 2 h_{22} h_{32} & h_{32}^{2} \\
h_{13}^{2}+h_{23}^{2} & 2 h_{13} h_{33} & 2 h_{23} h_{33} & h_{33}^{2} \\
h_{14}^{2}+h_{24}^{2} & 2 h_{14} h_{34} & 2 h_{24} h_{34} & h_{34}^{2}
\end{array}\right] \\
& \mathbf{x}=z_{A}^{2}\left[\frac{1}{f^{2}}, \quad-\frac{x_{0}}{f^{2}}, \quad-\frac{y_{0}}{f^{2}}, \quad \frac{x_{0}^{2}}{f^{2}}+\frac{y_{0}^{2}}{f^{2}}+1\right]^{T}
\end{aligned}
$$

and $h_{j i}$ is $h_{j}$ in image $i$. The critical motions are according to equation (16) reduced to quadrics of the form

$$
\left[\begin{array}{llll}
a & 0 & c & 0 \\
0 & a & d & 0 \\
c & d & b & 0 \\
0 & 0 & 0 & 0
\end{array}\right]
$$

centered at $A$, where $a, b, c, d \in \mathbf{R}$. Other cases where a subset of the intrinsic parameters is known can be treated similarly.

\section{Experiments}

\subsection{Simulation}

In order to evaluate the calibration accuracy for motions close to being critical, an experiment on simulated data was 

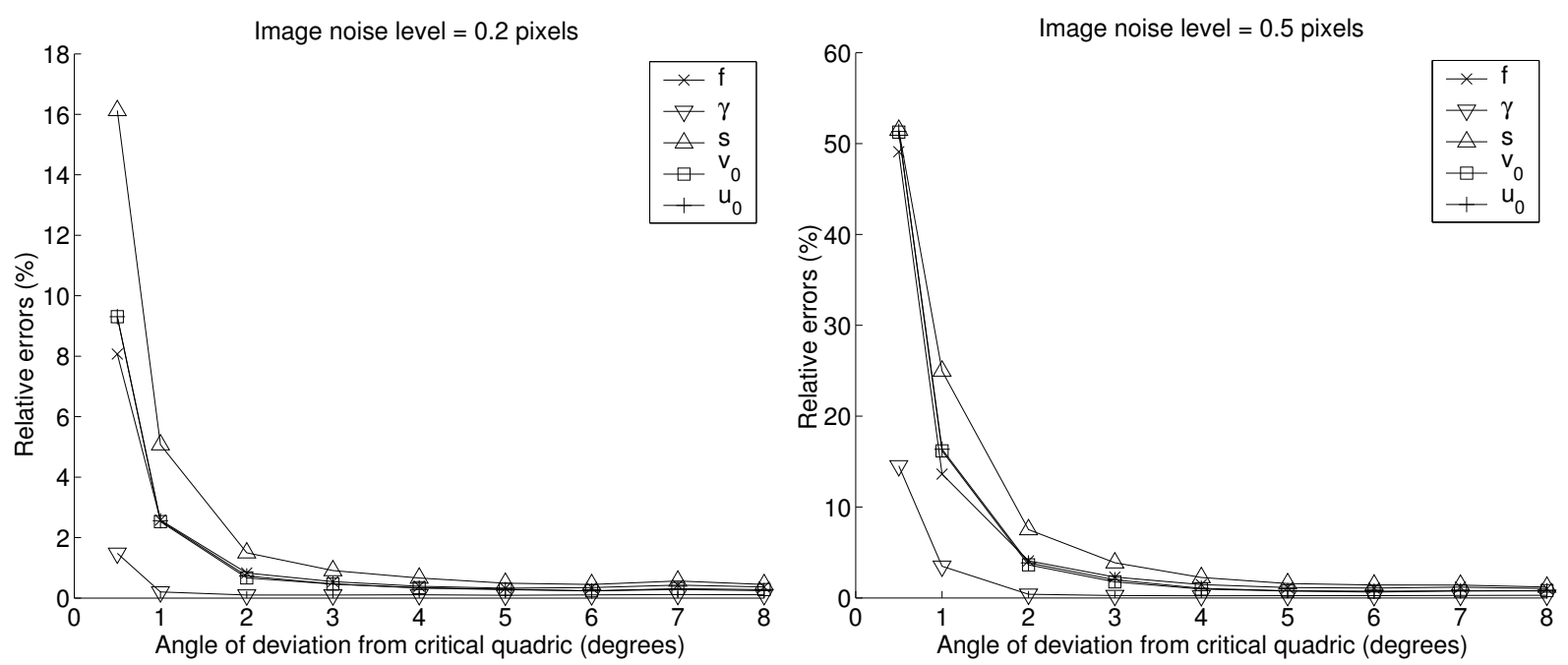

Figure 4. Calibration errors with respect to angle of deviation of the 1D objects from a critical quadric

performed. The simulated camera had $f=1000, \gamma=1$, $s=0$ and $\left(x_{0}, y_{0}\right)=(320,240)$. A stick of length $L=70$ with $\lambda_{A}=\lambda_{B}=0.5$ and fixed point $A=[0,35,150]^{T}$ was placed in 100 equally spaced positions on a critical cone. Gaussian noise with mean 0 and varying standard deviation was added to the angle between the stick and the axis of symmetry of the critical cone as illustrated in Figure 5. Gaussian noise with mean 0 and varying standard deviation was added to the obtained image points.

The calibration algorithm for the general case where all the intrinsic parameters are assumed to be unknown was used. We measure the relative accuracy of the focal length $|\Delta f / f|$ and the dimensionless quantities $|\Delta \gamma|,|\Delta s|$, $\left|\Delta u_{0} / f\right|$ and $\left|\Delta v_{0} / f\right|$ since errors in these contribute about equally to the overall geometric accuracy in scene reconstruction [11]. Results are given in Figure 4 for two different levels of image noise.

We note that the calibration results are very inaccurate for small angles of deviation from the critical surface as expected. The improvement in accuracy is very dramatic when increasing from close to $0^{\circ}$ deviation from the cone, to a few degrees. After around $5^{\circ}$ there is no big improvement and the results are quite good from this point on.

The fact that we get more accurate calibration results than in [14] is probably due to the stick being far from parallel to the optical axis of the camera. Since the endpoints of the image of the stick then are far apart, the results are less affected by noise.

\begin{tabular}{|c|c|c|}
\hline Errors (\%) & Sequence 1 & Sequence 2 \\
\hline$f$ & 1.3566 & 20.3945 \\
$\gamma$ & 1.5918 & 23.7308 \\
$s$ & 0.7971 & 1.1993 \\
$u_{0}$ & 4.6013 & 5.2164 \\
$v_{0}$ & 0.6743 & 3.7431 \\
\hline
\end{tabular}

Table 1. Experimental results for calibration from real data. In sequence 1 , the stick is moving randomly. In sequence 2 , the motion of the stick is such that it is close to a critical quadric surface

\subsection{Real Data Experiment}

To evaluate the sensitivity of the calibration algorithm in a real world scenario, a digital camera was calibrated using two separate image sequences containing images of a stick moving in two different patterns. The image resolution was $640 \times 480$ pixels. In the first sequence the stick was moved randomly. In the second sequence the stick was moved close to a critical surface, as illustrated in Figure 6. The camera was in both cases calibrated using the closed form solution for calibration from one dimensional objects given no knowledge of the intrinsic parameters, as described above. To be able to compare the results, the camera was also calibrated using the standard algorithm for calibration from planar patterns [13], including nonlinear minimization of the cameras intrinsic parameters from reprojection errors, resulting in a very precise calibration. The results are given 

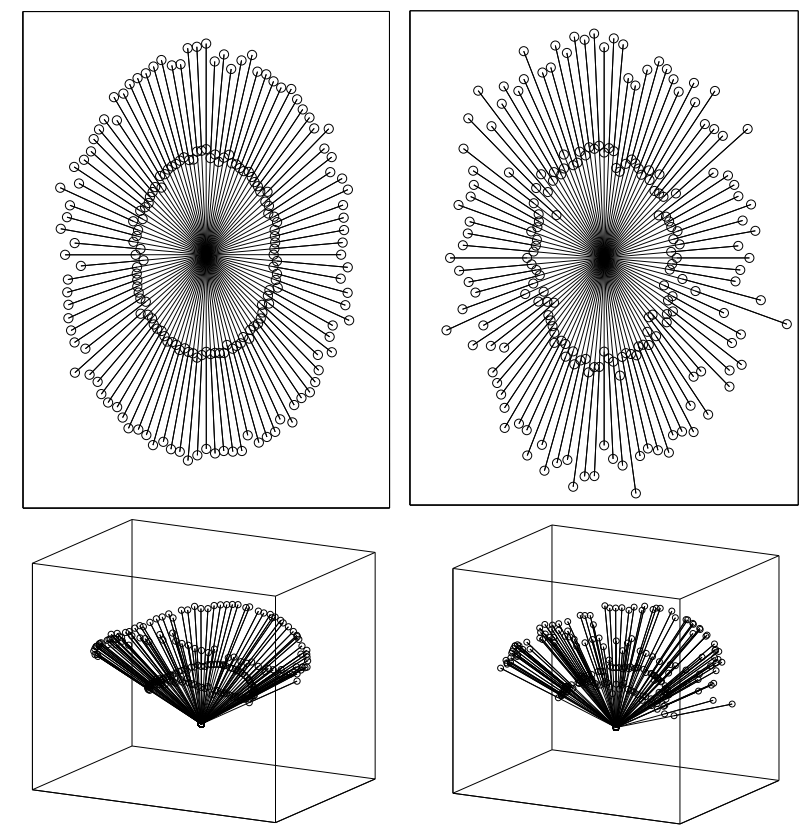

Figure 5. Simulation of sticks on a degenerate surface with added angular noise with a standard deviation of $2^{\circ}$ (left) and $5^{\circ}$ (right)

in Table 1, where the errors in the intrinsic parameters from each of the two calibration results are given with respect to the calibration result from the planar patterns. The errors from the sequence with the degenerate stick movement is generally much larger than for the random movement sequence, suggesting that close-to-critical motions of the stick has to be avoided in practice.

\section{Summary and Conclusions}

Based on a geometrical interpretation of the constraint used in camera calibration with one-dimensional objects, we have identified the critical motions where the calibration algorithm will fail. We have shown that constraints on the intrinsic parameters of the camera lead to simplified closed-form-solutions and a reduced set of critical motions, and also proposed some safe non-critical motions that will guarantee the success of the calibration algorithm in practice. A simulation and a real data experiment was performed to evaluate the calibration accuracy for motions close to being critical, showing the sensitivity of the algorithm to these motions.
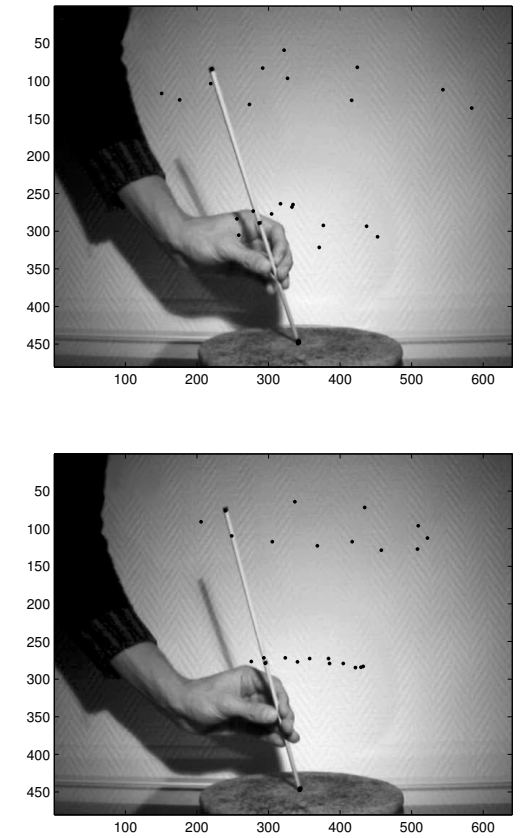

Figure 6. Two images from two image sequences, each consisting of 12 images. On each of the two images, tracked points from the entire sequence are superimposed. In sequence 1 , the stick is moving in a random fashion (top). In sequence 2, the motion of the stick is such that it is close to a critical quadric surface in each image (bottom)

\section{References}

[1] M. Armstrong, A. Zisserman, and P. Beardsley. Euclidean structure from uncalibrated images. In E. Hancock, editor, Proceedings of the 5th British Machine Vision Conference, York, England, volume 2, pages 509-518, September 1994.

[2] T. Buchanan. The twisted cubic and camera calibration. Computer Vision, Graphics and Image Processing, 42:130-132, 1988.

[3] R. Hartley and A. Zisserman. Multiple View Geometry in Computer Vision. Cambridge University Press, 2000.

[4] R. I. Hartley. Self-calibration from multiple views with a rotating camera. In Proc. European Conf. on Computer Vision, pages 471-478, Stockholm, Sweden, 1994. 
[5] A. Heyden and K. Åström. Flexible calibration: Minimal cases for auto-calibration. In Proc. Int. Conf. on Computer Vision, pages 350-355, Kerkyra, Greece, 1999.

[6] A. Heyden and K. Åström. Euclidean Reconstruction from Image Sequences with Varying and Unknown Focal Length and Principal Point. In Proc. Conf. Computer Vision and Pattern Recognition, pages 438-443, San Juan, Puerto Rico, 1997.

[7] S. J. Maybank and O. D. Faugeras. A theory of self calibration of a moving camera. Int. Journal of Computer Vision, 8(2):123-151, 1992.

[8] M. Pollefeys, L. Van Gool, and M. Proesmans. Euclidean $3 \mathrm{~d}$ reconstruction from image sequences with variable focal lengths. In Proc. European Conf. on Computer Vision, pages 31-42, Cambridge, UK, 1996.

[9] P. Sturm. Critical motion sequences for monocular self-calibration and uncalibrated Euclidean reconstruction. In Proc. Conf. Computer Vision and Pattern Recognition, pages 1100-1105, San Juan, Puerto Rico, 1997.

[10] P. Sturm and S. Maybank. On plane-based camera calibration: A general algorithm, singularities, applications. In Proceedings of the Conference on Computer Vision and Pattern Recognition, Fort Collins, Colorado, USA, pages 432-437, June 1999.

[11] B. Triggs. Autocalibration from planar scenes. In Proc. European Conf. on Computer Vision, volume I, pages 89-105, Freiburg, Germany, 1998.

[12] R.Y. Tsai. A versatile camera calibration technique for high-accuracy 3D machine vision metrology using off-the-shelf TV cameras and lenses. IEEE Journal of Robotics and Automation, 3(4):323-344, August 1987.

[13] Z. Zhang. A flexible new technique for camera calibration. IEEE Trans. Pattern Analysis and Machine Intelligence, 22(11):1330-1334, 2000.

[14] Z. Zhang. Camera calibration with one-dimensional objects. IEEE Trans. Pattern Analysis and Machine Intelligence, 26(7):892-899, 2004. 